\title{
Identification comparée des sables vacuolaires à terre et en mer
}

\author{
A. Baltzer ${ }^{1}$, A. Stépanian ${ }^{2}$, J. Owono ${ }^{3}$, B. Tessier ${ }^{4}$ E., Chaumillon ${ }^{5}$ \\ ${ }^{1}$ Maître de Conférences, ${ }^{2-3}$ Etudiants, ${ }^{4}$ Chercheur CNRS, \\ Université de Caen, Morphodynamique Continentale et Côtière (M2C), \\ 24 rue des Tilleuls,14000 CAEN.Tel :0231565745.baltzer@geos.unicaen.fr \\ ${ }^{5}$ Maître de Conférences. Université de La Rochelle, CLDG \\ Avenue Michel Crépeau, 17042 LA ROCHELLE
}

Résumé

Les sables vacuolaires sont des sables de plages soumises à l'action des marées, qui présentent une structure de type éponge en coupe verticale. Des bulles d'air sont piégées dans la partie superficielle du sable jusqu'à une profondeur d'environ 30 $60 \mathrm{~cm}$. Identifiés pour la première fois en conditions immergées sur des profils sismiques très haute résolution obtenus avec un boomer « Seistec », les résultats sont comparés à ceux acquis sur la plage d'Omaha Beach. Trois paramètres, décrits dans la littérature, conditionnent l'établissement des sables vacuolaires : la granulométrie, la teneur en eau, l'existence d'un marnage sur une pente inférieure à $8^{\circ}$. Nous mettons en évidence un quatrième facteur, une morphologie spécifique, favorisant appréciablement le piégeage des bulles d'air.

\section{Abstract}

Soft sands are due to a sponge like structure : the bubbles are formed by air trapped in sand when the area is flooded by the rising tide. This process affects the superficial sediments until a maximum depth of 30 to $60 \mathrm{~cm}$. Identified for the first time using a « Seistec » boomer, a very high resolution seismic tool, these data are compared to results acquired on Omaha Beach. In the literature, three factors conditioning soft sand occurrence are usually described: grain-size, water content, tidal variations on beach slope with a maximum dip of $8^{\circ}$. In this paper we outlight a fourth parameter: the local morphology which is able to highly improve the air bubble trapping.

\section{Introduction}

Les sables vacuolaires ont été décrits pour la première fois par Kindle (1936). Bien qu'ils soient présents sur la plupart des plages sous influence tidale, curieusement, très peu d'études existent à leur sujet, et les paramètres favorisant leur formation restent encore mal connus. Ne supportant aucune charge, ils peuvent pourtant constituer un facteur limitant à l'implantation d'ouvrages dans les secteurs côtiers 
les plus favorables à leur formation dont il convient par conséquent de cerner les mécanismes.

Une nouvelle approche pour l'étude de ces sables est présentée ici, grâce à un outil sismique de très haute résolution : un boomer de type «Seistec ». Ces données acoustiques acquises « en mer » sur le Banc du Bûcheron situé à l'embouchure du Fier d'Ars, au Nord de l'lle de Ré, sont comparées à des données (résistance à la pointe, teneur en eau, granulométrie) acquises « à terre " sur la plage d'Omaha Beach, en Normandie.

Les sables vacuolaires ou sables vésiculeux ou encore soft sands sont des sables de plage généralement trouvés en limite supérieure de la zone intertidale, dans lesquels sont emprisonnées des bulles d'air jusqu'à une profondeur de 30 à $60 \mathrm{~cm}$. Leur formation est limitée en profondeur par un horizon imperméable ou une nappe d'eau continue (Allen, 1982) et présentent, en coupe verticale, l'aspect d'une structure en éponge. Les trous ne sont pas verticaux, sauf en surface (Palmer, 1928), et leur diamètre peut varier de quelques millimètres à un maximum de $2 \mathrm{~cm}$. Le caractère malléable «mousseux» des sables vacuolaires tient au fait que la tension superficielle de l'eau interstitielle est suffisante pour maintenir les grains en place autour des cavités, mais n'est pas suffisante pour supporter un poids quelconque.

Les facteurs favorisant la formation des sables vacuolaires sont d'après la littérature, liés à la pente, à la taille des sables et à la teneur en eau. Une pente inférieure à $8^{\circ}$ en zone protégée du déferlement est requise (Emery, 1945), les zones à fort marnage offrant une plus grande surface de développement potentiel. Un sable bien trié favorise la formation de bulles d'air, l'homogénéité de la taille des grains empêchant le colmatage par les fines. Si la granulométrie est trop grosse, l'eau s'infiltre plus facilement, ne laissant pas de possibilité à l'air d'être piégé. Inversement, si la taille des grains est trop petite, l'air ne pourra pas s'introduire dans le sable : la perméabilité, via la granulométrie, contrôle ainsi la formation du sable vacuolaire (Kazanci et al., 1998). Enfin, la teneur en eau semble jouer un rôle non négligeable : un sable sec donne plus facilement lieu à une structure vacuolaire. Les dépôts de sable sec de haute plage favorisent donc ce processus, le volume d'air contenu dans les vides étant plus important que dans du sable humide.

\section{Les observations « à terre ": la Plage d'Omaha Beach}

La plage d'Omaha Beach, en Normandie, est soumise à un régime macrotidal, semidiurne, avec un marnage variant de 5 à $7 \mathrm{~m}$ en vives-eaux. Orientée Est-Ouest, elle subit les houles de secteur Nord et présente une large zone de swash. Sa morphologie montre une succession de 2 barres et 2 bâches du pied de dune vers la mer (Fig.1), pour une pente générale de $2^{\circ}$. Le sable possède une granulométrie unimodale centrée sur une médiane variant de 258 à $277 \mu \mathrm{m}$, regroupant $80 \%$ des 
grains. Il s'agit donc d'un sable moyen bien trié, caractéristique de la plage d'Omaha.

Afin de mettre en évidence l'existence de sable vacuolaire, et définir leur zone de formation préférentielle, deux séries de mesures (résistance à la pointe, teneur en eau, granulométrie) ont été réalisées à 4 jours d'intervalle, le 8 et le 12/10/2000, le long d'un même profil transversal de la plage (Fig.1).

Dans les deux cas, les sables vacuolaires sont localisés préférentiellement au sommet de la barre principale, sur son flanc doux, juste en bord de bâche, à $160 \mathrm{~m}$ et $150 \mathrm{~m}$ de distance par rapport au pied de dune. Ils présentent des résistances à la pointe variant de 0,2 à 0,5 , soit un rapport de 2 à 4 fois moins que du sable normal $(O, 8)$. Pour l'exemple du $8 / 10$, une deuxième formation de sable vacuolaire apparaît à l'extrémité de la barre de haut de plage, mais moins bien marquée. Le $12 / 10$, une trace de sable vacuolaire persiste à une distance également de $60 \mathrm{~m}$ par rapport au pied de dune. Le niveau maximum atteint par la marée, les 8 et 12/10 était de $1,57 \mathrm{~m}$ et $2,60 \mathrm{~m}$ respectivement.

Les teneurs en eau des échantillons prélevés dans ces sables vacuolaires, conformément à ce qui est décrit dans la littérature, présentent des valeurs faibles ( $w<10 \%)$, en comparaison des échantillons de sables normaux $(w>15-20 \%$ ).

\section{Les observations « en mer» : Le Banc du Bûcheron, Fier d'Ars - Ile de Ré}

Lors d'une campagne d'acquisition de données sismiques très haute résolution utilisant un Boomer de type «Seistec » dont la résolution verticale est de l'ordre de $25 \mathrm{~cm}, 25$ profils ont été réalisés à l'entrée du Fier d'Ars, au Nord de l'île de Ré, pour reconnaître, entre autres, la géométrie du Banc du Bûcheron. Ce Banc correspond à une flèche sableuse en partie intertidale formée, telle un plage, sous l'action de la houle à l'embouchure du Fier (Fig. 2A). Il découvre régulièrement à marée basse, le marnage moyen étant dans cette région de $4 \mathrm{~m}$. Sur plusieurs profils réalisés au travers du banc, une signature acoustique très particulière peut être observée (Fig. 3). Il s'agit d'une signature de type " point brillant» ou «bright spot $"$, reconnue et décrite habituellement dans la littérature dans des sédiments de milieux profonds (Hovland et Judd, 1988). Elle apparaît comme la succession d'un ou deux réflecteurs très marqués (noirs) intercalés avec un ou deux blancs acoustiques, ce qui traduit des différences d'impédance, donc de vitesse et de densité, très importantes. Cette signature reste très localisée, sans large extension latérale, d'où son nom de point brillant. Elle est interprétée comme indice de la présence de gaz dans le sédiment, diminuant ainsi brusquement l'impédance de la couche traversée par l'onde acoustique. La figure 2B montre la localisation des points brillants reconnus sur le banc du Bûcheron. Dans tous les cas, ils apparaissent sur des hauts morphologiques du banc. De plus, ils sont situés le plus souvent sur le flanc doux du banc, tourné vers la mer, fréquemment en surplomb d'un dénivelé, en particulier du chenal d'embouchure du Fier (Fig. 3, exemple profil Dsi09). Cette disposition est par conséquent comparable à celle des sables 


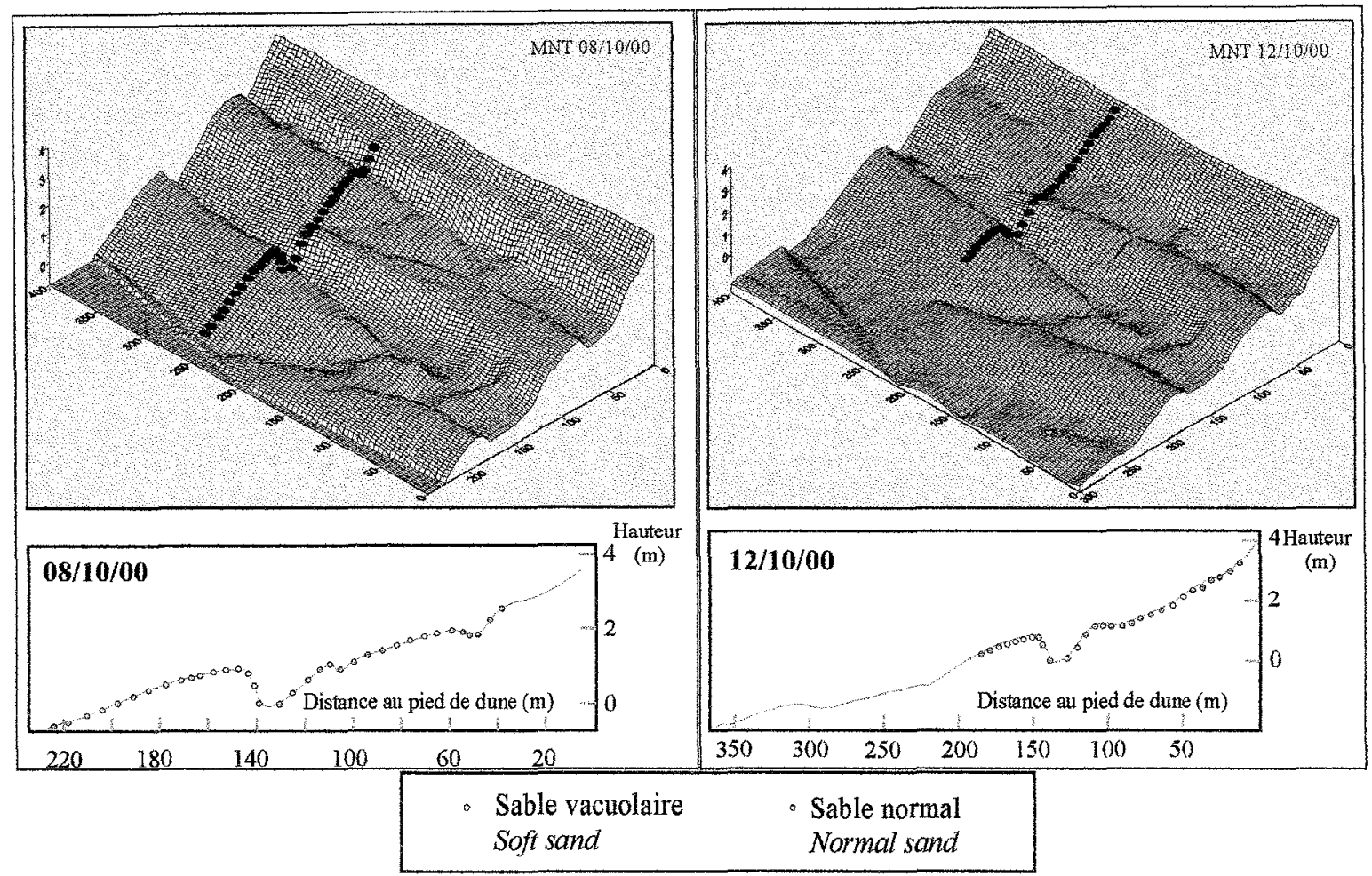

Figure 1 : Comparaison des données de résistance à la pointe sur la plage d'Omaha Beach acquises sur un même profil à deux dates différentes : le 8/10/2000 et le 12/10/2000. Comparison of two sets of penetration resistance data acquired on a same profile of Omaha Beach at two different dates: the 8/10/2000 and the 12/10/2000. 
vacuolaires observés sur le système de barres et de bâches de la plage d'Omaha Beach. A noter que le sable du banc est un sable moyen (médiane $330 \mathrm{~mm}$ ) bien trié, donc également comparable à celui d'Omaha. Compte tenu de la position intertidale du banc, nous avons pu vérifier à marée basse que les points brillants correspondent en effet à des sables vacuolaires, l'air piégé dans le sédiment provoquant une signature acoustique attribuée ordinairement à la présence gaz.

\section{Discussion : mécanismes de mise en place des sables vacuolaires}

La formation des sables vacuolaires se fait généralement sous l'action de la marée. Différents mécanismes peuvent cependant être distingués suivant la position du sable sur l'estran : sous l'action du swash en bas de plage, ou sous l'action de la marée en haute plage où sont stockés des sable secs.

Nos deux exemples illustrent ces mécanismes : les deux zones découvrent à marée basse puis sont ennoyées à marée haute. Lors du backswash, des bulles d'air traversent la surface du sédiment, puis grossissent en s'associant, suivant l'épaisseur du film d'eau et la réorganisation du sable. Ces bulles piégées peuvent être millimétriques, de la taille des grains de sables ou centimétriques (alors plutôt de forme ovoïde ou elliptique). Puis des tubules apparaissent, petits cylindres verticaux pouvant atteindre plusieurs centimètres de longueur. Quand l'eau du swash s'est finalement retirée, un sable vacuolaire reste en place, les tubules étant préservées dans le sédiment encore humide. Le sable comporte ainsi des bulles d'air qui se déplacent à mesure que le sédiment se gorge d'eau lors de la marée montante. Or l'eau progresse plus vite à la surface du sédiment qu'au sein même du sédiment. La couche humide superficielle, maintenue en surface par le pouvoir de rétention du sable, retarde l'évacuation de l'air, et des bulles d'air (donc de gaz) sont piégées dans le sable. Ainsi, si la marée est trop rapide pour que l'air retenu dans les interstices du sable puisse s'échapper, ce dernier conservera des bulles en sub-surface. Au jusant, une partie de l'eau emprisonnée dans le sédiment s'évapore ou se trouve drainée, augmentant encore le nombre d'inclusions gazeuses dans le sable. La prochaine marée détruira le structure en éponge dès que la première vague atteindra ce sédiment, pendant que dans le même temps le processus recommencera pour former un nouveau sable vacuolaire (Hoyt et Henry, 1964).

Les trois paramètres évoqués dans la littérature, une plage à faible pente sous l'influence d'un important marnage, une granulométrie très homogène ( $80 \%$ des grains autour des médianes de 270 microns (Omaha Beach) et 320 microns (Banc du Bûcheron), une teneur en eau faible ( $<10 \%$ pour Omaha) sont vérifiés dans nos deux exemples. Par contre l'existence d'un $4{ }^{\text {ème }}$ paramètre semble particulièrement importante pour l'établissement des sables vacuolaires : la morphologie locale. En effet dans chacun des cas, l'apparition des sables vacuolaires se fait sur un haut topographique situé à proximité immédiate d'un bas topographique : un sommet de barre en bordure de bâche dans le cas de la plage d'Omaha Beach, les parties hautes 


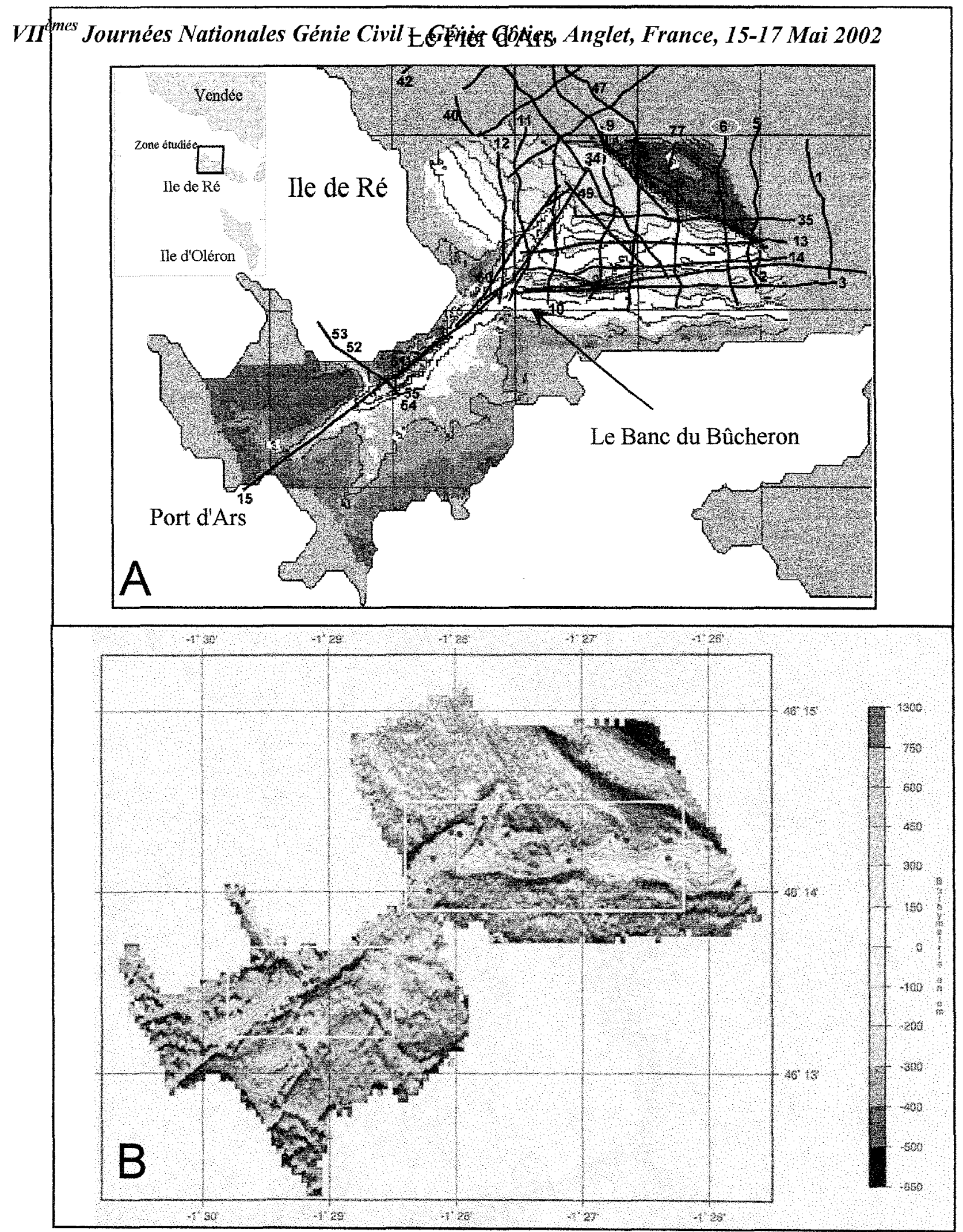

Figure 2: Le Banc du Bûcheron, embouchure du Fier d'Ars, He de Ré. (A) : Localisation des profils sismiques Seistec acquis sur le banc. Les numéros de profils entourés correspondent aux extraits montrés sur la figure 3 ; (B): Position des "points brillants" sur le banc (cercles noirs).

The Bucheron Bank, Fier d'Ars mouth, lle of Ré. (A): Localisation of acquired seismic Seistec profiles on the Bank. White circles show the selected profiles given on the figure 3. (B): "Bright spots" position (black circles) on the bank 

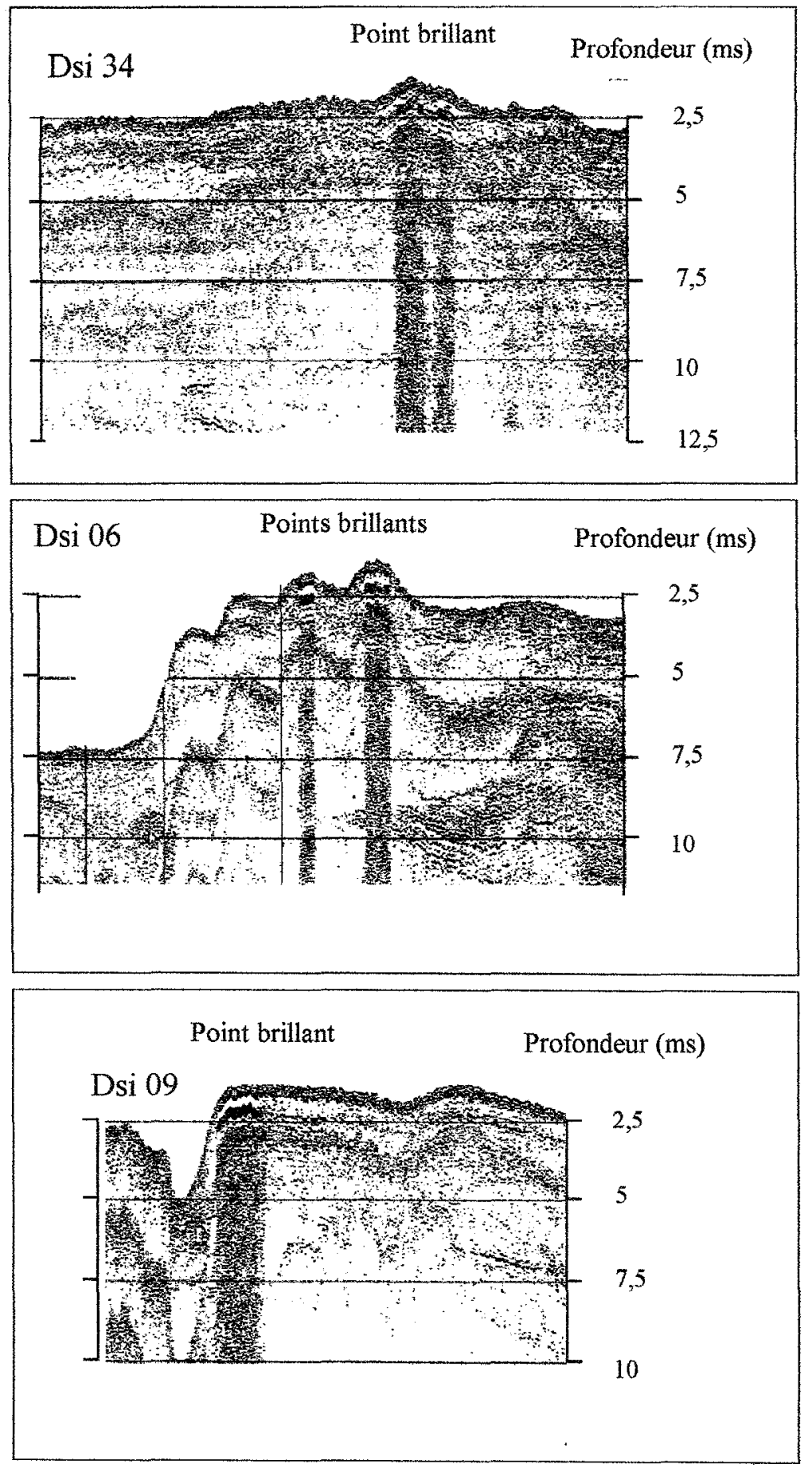

Figure 3 : Profils sismiques boomer "Seistec" sur Le Banc du Bucheron. La profondeur d'eau est donnée en ms.

Examples of boomer "Seistec" profiles on the Bucheron Bank. Water depth is indicated in ms. 
du Banc du Bûcheron, soit en bordure du chenal d'évacuation du Fier (Fig. 3 - Dsi 09) soit au sein de la succession de creux et bosses modelant le sommet du banc (Fig. 3- Dsi 06 et Dsi 34). Cette configuration morphologique conduit la marée montante à encercler le haut topographique, empêchant ainsi l'air de s'échapper « en avant » au fur et à mesure que l'eau monte. L'air est alors piégé dans le haut topographique, puis finalement emprisonné par la pellicule d'eau déposée par la première vague qui le franchit; les conditions optimum de piégeage de l'air sont réunies lorsque la vitesse du flot est maximum, c'est à dire à mi-marée, permettant une saturation rapide des couches inférieures.

\section{Conclusion}

Cette nouvelle approche d'étude des sables vacuolaires, développée à partir de l'analyse d'imagerie sismique très haute résolution obtenue avec un boomer de type "Seistec ", nous permet de mettre en évidence leur existence en conditions immergées. Leur signature acoustique est similaire à celles des points brillants communément décrits dans les faciès sédimentaires profonds où existent des indices de gaz. La comparaison de deux sets de données, l'un acquis sur la plage d'Omaha Beach, en conditions émergées, et l'autre sur le banc du Bûcheron en conditions immergées, nous permet de dégager un nouveau facteur déterminant pour la mise en place de ces sables. Ainsi non seulement la granulométrie, la teneur en eau, et le marnage constituent des paramètres essentiels, mais également la topographie locale. Un haut topographique situé à proximité immédiate d'un dénivelé permettra de piéger efficacement l'air contenu dans le sable en l'empêchant d'être évacué au fur et à mesure de la progression de la marée. Ainsi l'identification de telles configurations, favorisant la création de sables vacuolaires, devrait permettre d'éviter le choix de tels sites pour l'implantation d'ouvrages côtiers, étant donné leurs caractéristiques géotechniques instables.

\section{Remerciements}

Les auteurs tiennent à remercier le PNEC ART7 pour le financement de l'étude sur Omaha Beach ainsi que l'Université de La Rochelle pour le financement de la mission Désirée.

\section{Références}

ALLEN J.R.J., 1982. Sedimentary structures : their character and physical basis, Vol.2, Elsevier.

EMERY K.O., 1945. Entrapment of air in beach sand, J. Sedim. Petrol., Vol. 15, pp 39-49.

HOYT J.H. \& HENRY V.J., 1964. Development and geologic significance of soft beach sand, Sedimentology, 3, pp 44-51. 
HOVLAND M., JUDD A.G., 1988. In : Seabed Pockmarks and seepages, impact on Geology, Biology and the marine environment. Ed : Graham and Trotman, London, pp 28-30.

KAZANCI N., ILERI O., VAROL B., ERGIN M., 1998. On the significance of small scale and short live air escape structures for the destruction of primary sedimentary lamination on the Colakly beach deposits, Gulf of Antalaya, Turkey (Eastern Mediterranean), Estuarine Coastal and Shelf Science, 47, pp 181-190.

KINDLE E.M., 1936. Dominant factors in the formation of firm and soft sand beaches, J. Sedim. Petrol., Vol 6, Nº1, pp 16-22.

PALMER R.H., 1928. Sand holes of the strand, Journ. Sed. Petrol. Vol. 10, pp 176180. 